\title{
A New Chemical Method of Surface-Treatment of Kevlar Fiber for Composites with Epoxy Resin
}

\author{
Dedicated to the Memory of the late Professor Ichiro Sakurada \\ M. TAKayanagi, S. Ueta, W.-Y. LeI, ${ }^{*}$ and K. KogA \\ Department of Industrial Chemistry, Kyushu Sangyo University, \\ Matsukadai, Fukuoka 813, Japan
}

(Received January 10, 1987)

\begin{abstract}
It was previously found that sodium hydride and dimethyl sulfoxide (DMSO) form sodium methylsulfinylcarbanion, which ionizes the proton of amide group of aramid molecules to dissolve them homogeneously in DMSO. Metalated poly( $p$-phenylene terephthalamide) reacted with alkyl halides and epoxy compounds. By utilizing this reaction, the surface layer of Kevlar fibers was partly decorated by functional groups. The interfacial adhesion between fiber surface and epoxy-amine resin was evaluated by a pull-out test of monofilament. Kevlar filament surfacemodified by polyfunctional epoxy resin was proven to give the highest adhesive strength. ESCA spectra supported an enrichment of epoxy on fiber surface. SEM micrographs of pulled out filament surface also suggested increased adhesion by epoxy decoration. The bending modulus of the composite of Kevlar cloth/epoxy-amine resin was increased by epoxy decoration and especially the T-peel strength of laminates with epoxy was remarkably increased. SEM micrographs revealed that the surface layer of Kevlar fibers was drastically damaged. This fact tells us that the ultimate adhesion has been achieved by our chemical method to the limit.

KEY WORDS Kevlar / Epoxy Resin / Composite Material / Pull-Out Test / Interfacial Adhesion / Bending Modulus / T-Peel Strength / Fracture /
\end{abstract}

One of the most important application of Kevlar aramid fibers is to use as fiber reinforcement in composite materials. Kevlar 49 was manufactured by du Pont Company mainly to use for the composite materials, having the characteristics of ultrahigh strength of 2.8 $\mathrm{GPa}$ and modulus of $130 \mathrm{GPa}$. Matrix resin used for Kevlar composite is usually epoxyamine resin. It is a general principle for the composite to use a ductile matrix to distribute the applied external force uniformly to individual fibers, resulting in homogeneous stress distribution, and to impede and deflect the crack growth at each interfacial boundary between fiber surface and matrix resin. For realizing this principle most efficiently, strong interfacial adhesion is a necessary condition for exhibiting full strength and modulus of reinforcing filaments. In a case of Kevlar fiber, such an efficient method of surface treatment has not yet been reported as seen in a silane coupling reagent applied to glass fibers. ${ }^{1} \mathrm{~A}$ few papers $^{2}$ proposed some methods of surfacetreatment of Kevlar which were not proven to be definitely effective.

This paper proposes a new chemical method, with which the skin layer of Kevlar fiber was broken when the composite cloth was peeled, suggesting the arrival at the highest adhesive strength. Epoxy resin is examplified as a matrix resin in this paper, but the principle for improving the interfacial adhesion pro-

\footnotetext{
* On leave from the North Western Technical University, Xyan, China.
} 
posed here was proven to be effective in the cases of other matrix resins. In our studies, a pull-out test of Kevlar monofilament from a layer of matrix resin was found to be effective in selection of the appropriate reagent for surface-treatment of fibers. Testing of the mechanical properties of the composites showed a good correspondence to the results evaluated by the pull-out test.

\section{EXPERIMENTAL}

\section{Principle of Surface Treatment of Kevlar 49}

The basic principle for increasing interfacial adhesion between Kevlar and a matrix resin is in that the functional groups capable of chemical reaction with matrix resin introduced on the Kevlar filament surface are expected to be effective from the chemical view point and the introduction of surface layer through a swelling reagent will be desirable for the adhesion due to the anchoring of matrix resin from the physical view point.

One of the authors ${ }^{3}$ reported in a previous paper that the reagent prepared by reaction of sodium hydride with dimethyl sulfoxide (DMSO) giving a solution of sodium methylsulfinylcarbanion in DMSO dissolved poly $(p$ phenylene terephthalamide) (PPTA) in inactive gas atmosphere, and that the polyanion of metalated PPTA dissolved in DMSO was able to react with various aliphatic and arylalkyl halides, giving $N$-substituted PPTA with almost $100 \mathrm{~mol} \%$ of yield. The reaction scheme is expressed as follows.

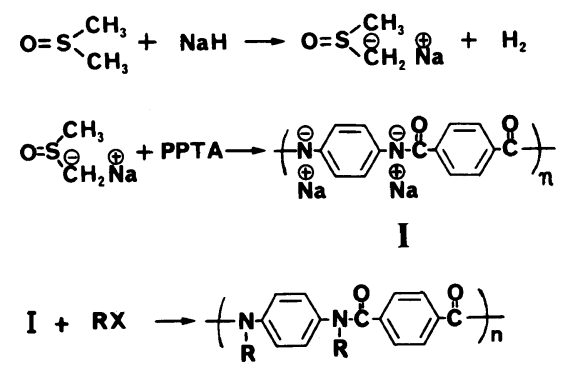

$\mathrm{R}$ : alkyl and arylalkyl groups
Reaction 2 gives a deep red colored, transparent solution and the reaction 3 proceeds homogeneously in a DMSO solution.

Substitution of amide proton of PPTA with carboxymethyl group was conducted by using monobromoacetic acid. ${ }^{4}$ The yield was about $50 \mathrm{~mol} \%$ substitution and the product was partially crystalline. After this method, epichlorohydrin and polyfunctional epoxy in absence of amine hardener were further adopted to provide useful functional groups to PPTA molecule.

As the reaction 2 dissolves aramid fibers completely after a long time, it was necessary to limit the extent of reaction to the surface layer thickness by controlling the reaction time. Surface-metalated Kevlar was transferred into another reaction bath to be substituted with desired functional groups. For monobromoacetic acid, $\mathrm{R}$ in equation 3 is $-\mathrm{CH}_{2} \mathrm{COOH}^{4}$ and for epichlorohydrin $\mathrm{R}$ is $-\mathrm{CH}_{2}-\underset{\mathrm{O}}{\mathrm{C}}-\mathrm{CH}_{2}$ and/or

$\left[\mathrm{CH}_{2} \mathrm{CH}\left(\mathrm{CH}_{2} \mathrm{Cl}\right)-\mathrm{O}_{m} \mathrm{H}^{5}\right.$ Nitrogen atom with negative charge in metalated PPTA attacks the primary carbon atom of epoxy group with nucleophilic substitution reaction. ${ }^{6}$ The reaction was confirmed by using phenyl glycidyl ether (PGE) as a model compound of epoxy resin. ${ }^{5}$ Elemental analysis gave $3 \mathrm{~mol}$ of PGE per one mol of repeating unit of PPTA. IR spectra showed 2940 and $2880 \mathrm{~cm}^{-1}$ of C-H of PGE and amide I, II, and III bands of PPTA at the same time. Thus, polyfunctional epoxy resin is convinced to partially substitute the proton on nitrogen atom of PPTA, which is expected to react with the matrix epoxyamine resin. Identification of the reaction products with IR and elemental analysis was made on the samples prepared by homogeneous reaction. ESCA spectra of surfacemodified Kevlar were measured and the composition of the surface was evaluated by referring to the calibration curves prepared for the homogeneous reaction products. 


\section{Surface Treatment of Kevlar 49}

Kevlar 49 as-received was washed with methylethylketone and dried at $100^{\circ} \mathrm{C}$ for $2 \mathrm{~h}$ (Sample A). For surface-metalation, $150 \mathrm{ml}$ of DMSO and $0.07 \mathrm{~g}$ of sodium hydride were reacted for $30 \mathrm{~min}$ at $30^{\circ} \mathrm{C}$ and for $40 \mathrm{~min}$ at $70^{\circ} \mathrm{C}$ in a nitrogen atmosphere. After cooling to $30^{\circ} \mathrm{C}$, Kevlar 49 fibers were immersed into the metalation bath and reacted for $10 \mathrm{~min}$. A part of skin layer of Kevlar was attacked by the reagent. A partially metalated skin layer of fiber swollen by DMSO was reacted by epichlorohydrin (Sample B) or by monobromoacetic acid (Sample C).

Epoxy resin was bisphenol-A type liquid epoxy, DER 383 of Dow Chemical Company with 176-186 of epoxy equivalent, molecular structure of which is approximately represented by

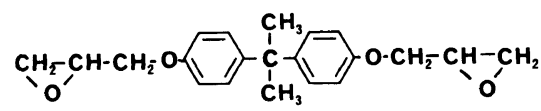

II

Amine hardener of epoxy resin was triethylene tetramine (TETA), which was used only for hardening the matrix resin in fabricating a composite. II was dissolved in DMSO, to which the surface-metalated Kevlar fibers taken out from a DMSO metalation bath were added. After reaction, excess epoxy was removed from treated Kevlar by acetone and dried at $50^{\circ} \mathrm{C}$ (Sample D).

\section{Surface Characterization of Kevlar 49 Fila- ment}

To ascertain the introduction of epoxy group on the Kevlar surface and evaluate the degree of substitution, ESCA spectra were taken on a sheet-like bundle of Kevlar fibers surface-modified by epoxy II. For this purpose, a series of powder samples of PPTA grafted with epoxy (PPTA-g-epoxy) at various degrees were prepared by homogeneous reaction on which the elemental analysis and the measurements of ESCA spectra were made.
First the calibration curves for the ratios of $\mathrm{C} / \mathrm{N}$ and $\mathrm{O} / \mathrm{N}$ were drawn by plotting both of the ratios determined by ESCA to those determined by elemental analysis. The calibration curves for the homogeneous samples are expected to give a linear relationship with slope of unity if the surface and inside compositions are the same. As mentioned later, the relation showed a steeper slope than unity. The nitrogen content determined by elemental analysis provides the average degree of grafting with epoxy II in $\mathrm{mol}^{\circ} \%$ per mol of repeating unit of PPTA. From the ratios of $\mathrm{C} / \mathrm{N}$ and $\mathrm{O} / \mathrm{N}$ of fibers, the compositions of surface layer of Kevlar modified by epoxy were evaluated based on the calibration curves and the average degree of grafting in the surface layer was evaluated.

\section{Evaluation of Interfacial Adhesion by Pull-Out Test}

Kevlar or surface-modified Kevlar monofilament with radius $6 \mu \mathrm{m}$ was immersed in a liquid epoxy resin blended with a hardener (TETA). The blend ratio was 176 of epoxy equivalent to 24.3 of amine-hydrogen equivalent. The immersion length was less than $1 \mathrm{~mm}$ and heat-treated at $100^{\circ} \mathrm{C}$ for $2 \mathrm{~h}$. Figure 1 shows the layout of testing equipment. The rate of displacement of the clamp was $5 \mathrm{~mm} \mathrm{~min}^{-1}$ for $35 \mathrm{~mm}$ of filament length, using a tensile tester, Tensilon of Toyo Baldwin Company. The load-time (or displacement) curve arrived at the maximum value when the monofilament was pulled out or fractured. The maximum tensile stress, $\sigma$, was plotted against

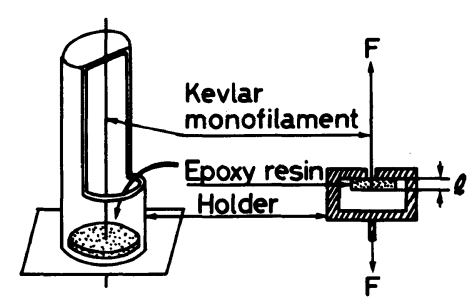

Figure 1. Layout of pull-out test of monofilament from epoxy resin. 
immersion length, $l$. Balance of force gives eq 4.

$$
\pi r^{2} \sigma=2 \pi r l \tau_{i}
$$

where $\sigma$ is tensile stress and $\tau_{i}$ is shear stress generated in interfacial layer. At the critical immersion length, $l_{c}$, the monofilament fractures at $\sigma_{f}$ and the shear stress amounts to $\tau_{i f}$. Then, eq 5 holds.

$$
\sigma_{f}=2 \tau_{i f} l_{c} / r
$$

The actual data of $\sigma$ and $l$ did not show a linear relation, in which case the apparent value of $\tau_{i f}$ evaluated by eq 5 was assumed to be the measure of interfacial adhesion.

SEM observation was made to inspect the damage of skin layer of Kevlar monofilament caused by adhesion of the matrix resin during the pull-out process.

\section{Preparation and Mechanical Testing of Kevlar Cloth/Epoxy Composites}

Kevlar cloth with plain weave texture was composed of 17 yarns $/ 25 \mathrm{~mm}$ width (1140 deniers/yarn). The laminate of Kevlar cloths was prepared according to JIS K6919. Four sheets of surface-cleaned Kevlar cloth or surface-treated Kevlar cloth were impregnated into the matrix resin, which was prepared by blending an epoxy of DER 383 and a hardener of TETA with equivalent composition of $13.5 \mathrm{pts}$ TETA to $100 \mathrm{pts}$ of DER 383. The resin content in the composite was adjusted to $35-40 \%$.

The surface-treatment of Kevlar cloth was conducted as follows: the cloths were dipped in a metalation bath prepared by $0.07 \mathrm{~g} \mathrm{NaH}$ and $150 \mathrm{ml}$ of DMSO at $30^{\circ} \mathrm{C}$ for $10 \mathrm{~min}$ and after taking out, the cloths were reacted with $10 \mathrm{~g}$ of epichlorohydrin dissolved in $100 \mathrm{ml}$ of DMSO (Sample B), with $10 \mathrm{~g}$ of monobromoacetic acid dissolved in $100 \mathrm{ml}$ of DMSO (Sample C) and with $10 \mathrm{~g}$ of DER 383 dissolved in $100 \mathrm{ml}$ of DMSO (Sample D). Reaction temperature was $50^{\circ} \mathrm{C}$ for $30 \mathrm{~min}$, the treated cloth was washed with acetone and dried at $50^{\circ} \mathrm{C}$ for
$30 \mathrm{~min}$.

The composite laminate was prepared in a vacuum bag under the load of $10 \mathrm{~kg}$ per $10 \times 15 \mathrm{~cm}^{2}$ of area. Precuring was conducted at $50^{\circ} \mathrm{C}$ for $1 \mathrm{~h}$ in a thermostat and postcured at $120^{\circ} \mathrm{C}$ for $2 \mathrm{~h}$ by using a hot plate. The specimen $1.0 \mathrm{~mm}$ thick, $15 \mathrm{~mm}$ wide and $25 \mathrm{~mm}$ long was cut out from the laminated sheet and the bending test was conducted with the jig of three-point support. Bending modulus and bending strength were evaluated according to the conventional formulae.

The T-peel test was conducted on the specimen $15 \mathrm{~mm}$ wide and $150 \mathrm{~mm}$ long using two sheets, in one end of which a Teflon sheet was inserted between both cloths to avoid the adhesion. By stretching unbonded cloth ends, the $T$-peel process was recorded on a chart.

SEM observation of the peeled surface is useful to judge the degree of interfacial adhesion based on the degree of surface damage of Kevlar filament.

\section{RESULTS AND DISCUSSION}

\section{Surface Characterization of Un-and Surface-}

\section{Treated Kevlar 49}

Figure 2 shows the comparison of ESCA spectra of (a) as-received and surface-cleaned Kevlar 49, (b) Kevlar 49 surface-modified by epoxy in absence of amine hardener, and (c) powder samples of PPTA-g-epoxy prepared by homogeneous reaction. The intensity of nitrogen peak is reduced by surface-modification, while the profiles of carbon and oxygen spectra of surface-modified Kevlar 49 are similar to those of the original one. More detailed inspection reveals that, in the carbon $1 \mathrm{~s}$ spectrum of PPTA-g-epoxy, the shoulder due to the ether carbon appears when the degree of grafting with epoxy exceeds about $100 \mathrm{~mol} \%$. The degree of grafting was determined by elemental analysis, which amounted to $105 \mathrm{~mol} \%$ for the sample shown by curve (c) in Figure 2. The ratios of $\mathrm{C} / \mathrm{N}$ and $\mathrm{O} / \mathrm{N}$ for Kevlar 49 are 12.5 and 5.0, respectively, and 


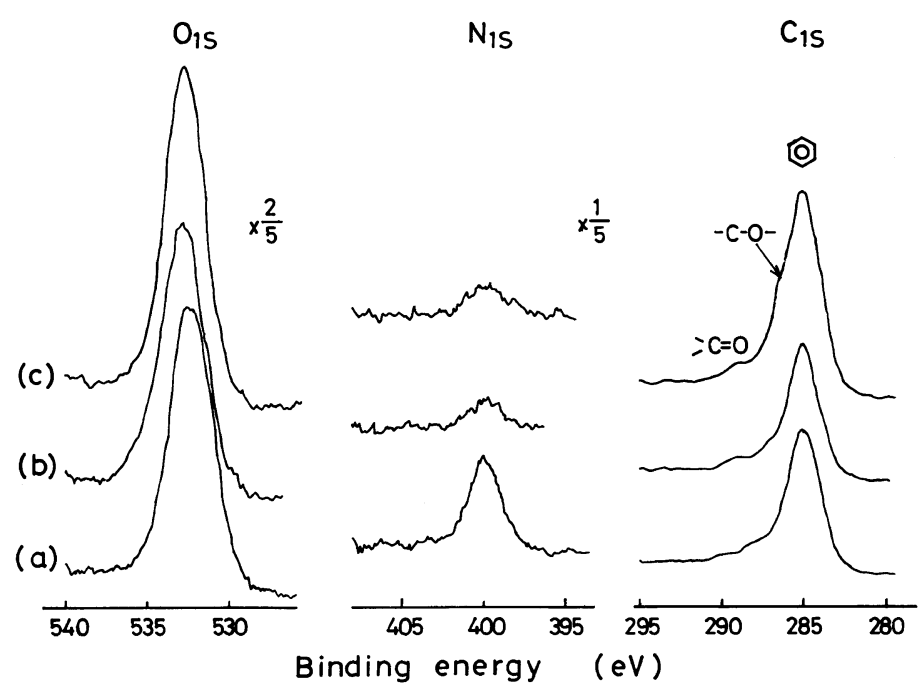

Figure 2. ESCA spectra of $\mathrm{O}_{1 \mathrm{~s}}, \mathrm{~N}_{1 \mathrm{~s}}$, and $\mathrm{C}_{1 \mathrm{~s}}$ for (a) as-received Kevlar 49, (b) Kevlar surface-modified by epoxy, and (c) powder sample of PPTA-g-epoxy prepared by homogeneous reaction $(105 \mathrm{~mol} \%$ of degree of substitution).

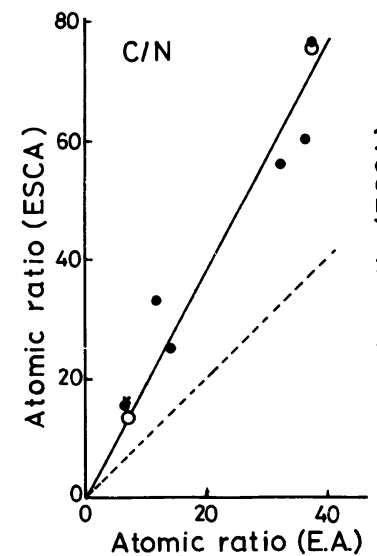

(a)

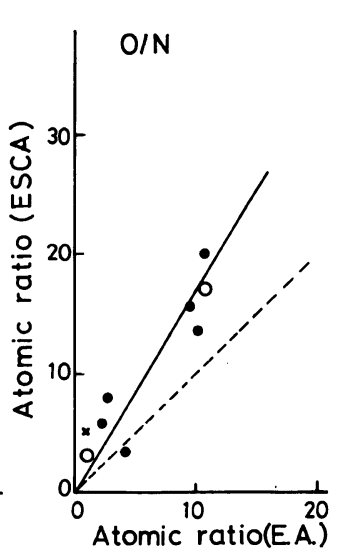

(b)
Figure 3. The atomic ratios determined by ESCA $v s$. those determined by elemental analysis (E.M.) for (a) $\mathrm{C} / \mathrm{N}$ and (b) $\mathrm{O} / \mathrm{N}$. Filled circles, powder samples; open circles, film; cross, surface-modified fibers.

those for surface-modified Kevlar are 35 and 15 , respectively.

Figures 3(a) and (b) are the relationships between the ratios of $\mathrm{C} / \mathrm{N}$ and $\mathrm{O} / \mathrm{N}$ determined by ESCA and those determined by the elemental analysis for the powder samples of PPTA-g-epoxy prepared by homogeneous re- action of metalated PPTA and epoxy in DMSO. From the ESCA data, the compositions of the utmost surface of Kevlar 49 fibers are estimated in atomic ratios. On the other hand, the elemental analysis provides the average degree of grafting of polyfunctional epoxy on PPTA trunk based on the nitrogen content. The slope of the relationship between the ratios determined by ESCA and elemental analysis should be unity on the same scales of both axes. However, as seen in Figure 3, the ratio determined by ESCA is greater than the ratio calculated by elemental analysis. The reason for it might be ascribed to the enhancement of epoxy groups on the utmost surface of the powder samples.

By utilizing the relationships given in Figures 3(a) and (b), we evaluated the degree of grafting of polyfunctional epoxy for the sample used in Figure 2(b), according to which the degree of grafting of epoxy on the utmost surface of Kevlar 49 is evaluated about $80 \mathrm{~mol} \%$. The result indicates that the surface of Kevlar 49 is chemically modified by polyfunctional epoxy compounds. 


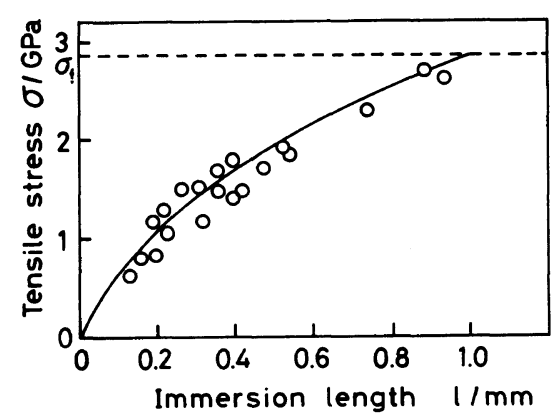

Figure 4. Tensile stress for pull-out vs. immersion length for surface-cleaned Kevlar 49 in pull-out test.

\section{Evaluation of Interfacial Adhesion by Pull-Out Test}

Figure 4 shows the relationship between the tensile stress at pull-out or fracture of filament and the immersion length for a surface-cleaned Kevlar 49. A horizontal broken line represents a level of stress at break of Kevlar 49. No linear relation can be obtained. A curve with decreasing slope finally crosses the broken line of stress level of fracture. The curve shows that the uniform sliding mechanisms at the interface layer do not hold but the local fracture at fiber surface or in matrix epoxy resin will take place at the maximum stress in stressstrain curve during pull-out test.

Another important point is an etching effect of metalation reagent for Kevlar 49 filament. Morphological observation clearly indicates the existence of skin structure, which is composed of highly oriented chains. The effect of etching with a metalation reagent on the filament strength was surveyed as a function of time. About $7 \%$ of decrease in strength was found after $10 \mathrm{~min}$.

Figure 5 summarizes the results of pull-out test for all kinds of surface-modified fibers from epoxy-amine resins. The critical length was determined at the crossing point of each curve with the line of $\sigma=\sigma_{f}$. The critical shear stress $\tau_{i f}$ was evaluated by substituting the $l_{c}$ value into eq 5 . The surface treatment providing the smallest $l_{c}$ and the largest $\tau_{i f}$ is the most efficient method for resisting to

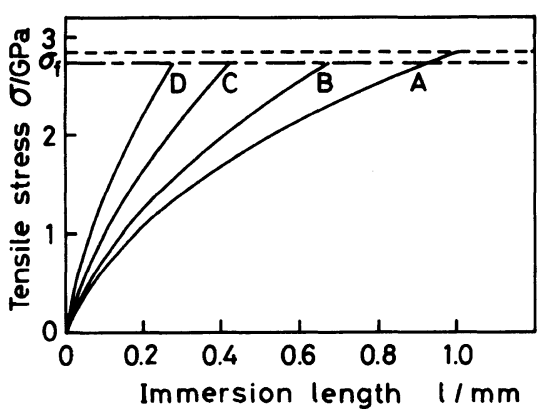

Figure 5. Tensile stress vs. immersion length in pullout test for various filaments. (A) As-received, (B) epichlorohydrin-reacted, (C) carboxymethylated, and (D) epoxy-reacted Kevlar 49 filaments. Broken and chain lines represent stress level of fracture.

Table I. Critical immersion length and interfacial shear strength calculated from pull-out test for various combinations of Kevlar and amine-epoxy resin

\begin{tabular}{|c|c|c|c|}
\hline \multirow{2}{*}{ Code } & \multirow{2}{*}{$\begin{array}{l}\text { Surface-treatment } \\
\text { of Kevlar } 49\end{array}$} & \multirow{2}{*}{$\frac{l_{c}}{\mathrm{~mm}}$} & \multirow{2}{*}{$\frac{\tau_{i f}}{\mathrm{MPa}}$} \\
\hline & & & \\
\hline A & Surface-cleaned & 1.03 & 8.96 \\
\hline B & $\begin{array}{l}\text { Epichlorohydrin- } \\
\text { reacted }\end{array}$ & 0.58 & 14.3 \\
\hline $\mathrm{C}$ & Carboxymethylated & 0.42 & 20.0 \\
\hline D & Epoxy-reacted & 0.27 & 31.5 \\
\hline
\end{tabular}

pull-out force. Table I lists the data of $l_{c}$ and $\tau_{i f}$ for various surface treatments. Referring to Figure 5 and the data of Table I, it is concluded that $N$-substitution with polyfunctional epoxy in Kevlar surface is most effective in pull-out test compared with other treatments.

The SEM micrographs of the surface of filament pulled out from the cured epoxyamine resin well corresponded to the results of pull-out test. In the case of $D$ in Figure 5, the surface of filament was distinctly roughened and fibrillated.

\section{Mechanical Properties and T-Peel Strength of Kevlar Cloth/Epoxy Composites \\ Table II summarizes the results of bending test of Kevlar cloth/epoxy composites with}


Table II. Comparison of bending tests for composites of Kevlar cloth and epoxy with various surface-treatments

\begin{tabular}{|c|c|c|c|c|}
\hline \multirow{2}{*}{ Code } & \multirow{2}{*}{$\begin{array}{l}\text { Surface-treatment } \\
\text { of Kevlar } 49 \text { cloth }\end{array}$} & $\begin{array}{l}\text { Yield } \\
\text { stress }\end{array}$ & \multirow{2}{*}{$\frac{\begin{array}{c}\text { Bending } \\
\text { modulus }\end{array}}{\mathrm{GPa}}$} & \multirow{2}{*}{$\begin{array}{c}\begin{array}{c}\text { Resin } \\
\text { content }\end{array} \\
\mathrm{wt} \%\end{array}$} \\
\hline & & $\mathrm{MPa}$ & & \\
\hline A & $\begin{array}{l}\text { As-received and } \\
\text { acetone-cleaned }\end{array}$ & 250 & 10.6 & 36 \\
\hline B & $\begin{array}{l}\text { Epichlorohydrin- } \\
\text { treated }\end{array}$ & 310 & 10.4 & 37 \\
\hline $\mathrm{C}$ & Carboxymethylated & 190 & 10.0 & 31 \\
\hline D & $\begin{array}{l}\text { Epoxy (DER 383)- } \\
\text { treated }\end{array}$ & 270 & 12.2 & 38 \\
\hline
\end{tabular}

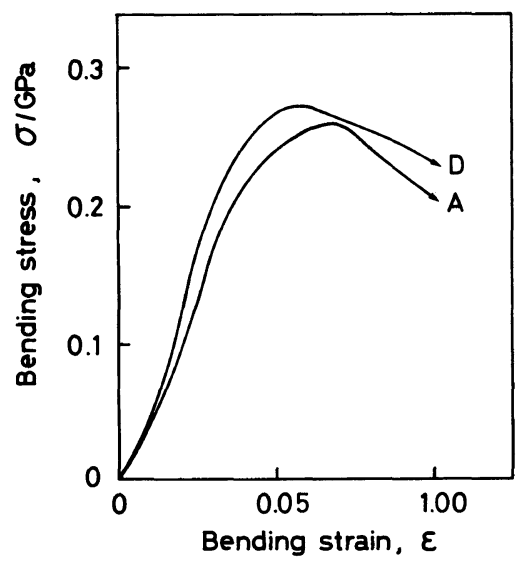

Figure 6. Bending stress $v s$. bending strain for Sample A and Sample D in Table II.

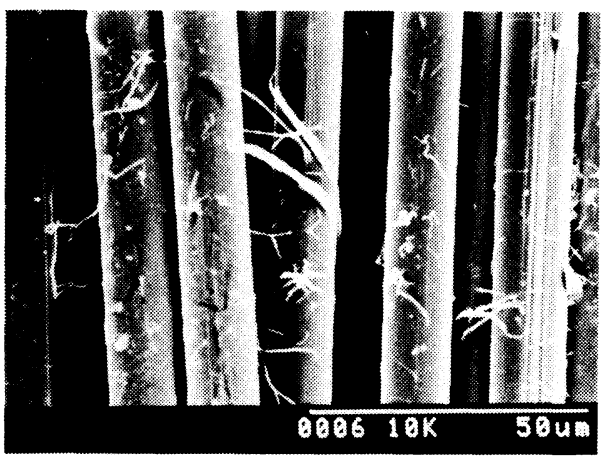

Sample A three point support.

Surface treatments of Kevlar cloth in Samples B and C are not so influential on bending modulus compared with that of Sample A. It should be noticed that Sample D of epoxy-treated Kevlar cloth composite shows the modulus increased by $15 \%$ compared with that of Sample A. Bending stressstrain curves of $A$ and $D$ are compared in Figure 6. An increased modulus of Sample D will be ascribed to the increased interfacial adhesion between Kevlar cloth surface and the epoxy-amine matrix resin, which is supported by the results of pull-out test of monofilament as shown in Table I. Yield stress of Sample D is also larger than that of Sample A.

Figure 7 shows the comparison of the proc-

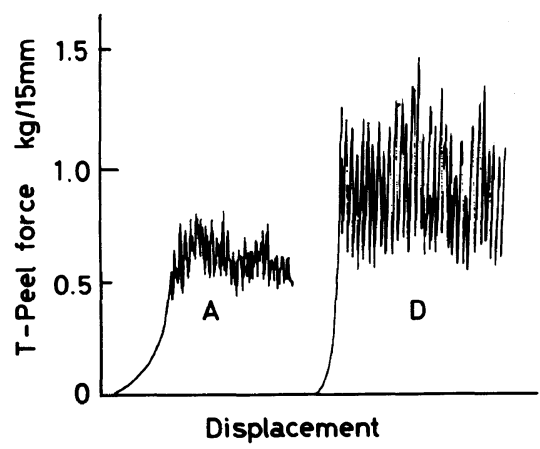

Figure 7. Peeling force $v s$. displacement during T-peel process for Sample A (left) and Sample D (right). A and $\mathrm{D}$ are the same as in Figure 6.

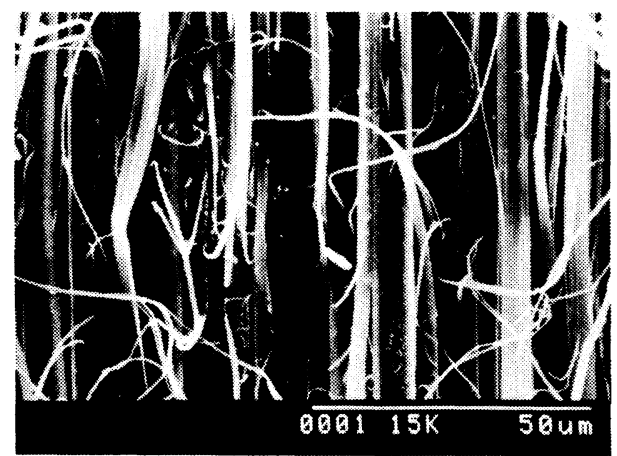

Sample D

Figure 8. SEM photographs of T-peeled surface of Sample A (left) and Sample D (right). A and D are the same as in Figure 6. 
ess of T-peel test in original chart, in which the left diagram is for Sample A and the right diagram is for Sample D. The fluctuation of peeling force during the stationary peeling process is due to the periodical contacts of yarns between two cloths laminated with epoxyamine resin. On the average, the T-peel force in Sample $D$ is about $0.9 \mathrm{kgf} / 15 \mathrm{~mm}$ width of cloth, while that of Sample A is about $0.6 \mathrm{kgf} / 15 \mathrm{~mm}$. The increase in $\mathrm{T}$-peel strength amounts to almost $50 \%$ for Sample D. The SEM photographs of peeled surface of Samples A and D are compared in Figure 8, in which the roughened surface of fibers in Sample D again supports the effectiveness of the surface treatment with polyfunctional epoxy resin. The skin layer of fibers is thoroughly broken, representing the limit of adhesion at which the filament surface itself can not endure the applied force.

Thus, it may be concluded that our method of surface treatment of Kevlar is conceived to be the best method so far proposed in improving the interfacial adhesion of Kevlar fibers in composites with epoxy, which reveals the full performance of Kevlar aramid fibers in a composite.

Acknowledgement. This work was partly supported in finances from the Asahi Chemical Industries Co. Use of ESCA is obliged to Professor T. Kajiyama, Kyushu University, to whom we express our thanks.

\section{REFERENCES}

1. D. Hull, "An Introduction to Composite Materials," Cambridge University Press, England, 1982, Section 3.4 .

2. Y. M. Mai and F. Castino, J. Mater. Sci., 19, 1638 (1984).

3. M. Takayanagi and T. Katayose, J. Polym. Sci., Polym. Chem. Ed., 19, 1133 (1981).

4. M. Takayanagi, T. Katayose, and T. Kajiyama, $J$. Appl. Polym. Sci., 27, 3903 (1982).

5. S. Ueta, Y. Nishihara, and M. Takayanagi, Bull. Fac. Eng., Kyushu Sangyo Univ., 22, 35 (1985).

6. M. Takayanagi and T. Katayose, J. Polym. Sci., Polym. Chem. Ed., 21, 31 (1983).

7. R. J. Morgan, C. O. Pruneda, and W. J. Steele, J. Polym. Sci., Polym. Phys. Ed., 21, 1757 (1983). 